\title{
Knowledge-Based Management and Reasoning on Cultural and Natural Touristic Routes
}

\author{
Evangelos A. Stathopoulos $\left.{ }^{(}\right)$, Alexandros Kokkalas, Eirini E. Mitsopoulou, \\ Athanasios T. Patenidis, Georgios Meditskos, Sotiris Diplaris, \\ Ioannis Paliokas, Stefanos Vrochidis, Konstantinos Votis, Dimitrios Tzovaras, \\ and Ioannis Kompatsiaris \\ Information Technologies Institute, Centre for Research \& Technology - Hellas, \\ Thessaloniki, Greece \\ \{estathop, akokkalas, emitsopou, apatenidis, gmeditsk, diplaris, ipaliokas, \\ stefanos, kvotis, dimitrios.tzovaras, ikom\}@iti.gr
}

\begin{abstract}
There is great potential in interdisciplinary traveling platforms mingling knowledge about cultural heritage aspects, such as places with schedules providing visits or even containing augmented reality features also, along with environmental concerns to enhance personalized tourist experience and tripping avocation. For an ontological framework to support and nominate trip detours of targeted interests according to end-users, it should incorporate and unify as much heterogeneous information, deriving either from web sources or wherever there are ubiquitously available such as sensors or open databases. A plethora of qualitatively diverse data along with adequate quantities of them escalate the contingent results in terms of conferring a plurality of relevant options which can be utterly manifested through involving axioms with rulebased reasoning functionalities upon properties considered to be irrelevant to each other at first glance. Thus, managing to import predefined concepts from other ontologies, such as temporality or spatiality, and combine them with new defined concepts to tourist assets, such as points of interest, results in novel meaningful relationships never established before. Apart from the utilization of pre-existent resources and logic towards automatic detouring suggestions, a wide-spectrum modeling enables a suitable problem statement relevant to the e-Tracer framework and comprehension of the issues, providing the opportunity of statistical analysis of knowledge when adequate amounts amassed.
\end{abstract}

Keywords: Ontologies - Reasoning - Semantically enriched geodata • Data homogenization $\cdot$ Route recommendation subsystem

\section{Introduction}

Vast amounts of data are effusive throughout every ecosystem. Gradually, the ability to effectively capture data for knowledge extraction has increased.

(C) IFIP International Federation for Information Processing 2020

Published by Springer Nature Switzerland AG 2020

I. Maglogiannis et al. (Eds.): AIAI 2020, IFIP AICT 583, pp. 355-367, 2020.

https://doi.org/10.1007/978-3-030-49161-1_30 
Digital agents tend to pseudomimic mental processes, such as deductive reasoning, intricate decision making, inferring general assumptions and so on. The chasm between data and knowledge is bridged by semantics, inserting and fusing contexts into otherwise meaningless data. Ultimately, the interest is not on the value itself but on its representation and meaning inside a system and its exploitation. Today, there are many methodologies followed towards knowledge design and manipulation; in that aspect, one can amalgamate disparate conceptions into a unified model. To achieve the wished level of homogeneity, the affinities of entities must become firmly established, the capitalization of which is the rapidity and deftness of knowledge elaboration to infer with logic as a basis.

In this paper we describe an ontology-based framework for capturing and interlinking assets of cultural and natural substance facilitating the formation of routes via the utilization of spatio-temporal rule-based reasoning. The ontological model encompasses and depicts every unique data genre present in the workflow of the platform: genres related to weather, topological formats expressing geometry and geospatiality (such as points in 3D space and routes), time formats expressing temporality, user profiling, Augmented Reality (AR) and hierarchical content categorization of places along with other information. That way, places might be discarded or included in a final route recommendation for the enduser. To complete this task, a systematic evaluation was performed to assess the abundance of our approach.

Our methodology starts by identifying each relevant entity tagged as coherent with the e-Tracer objectives, as described above. Thereinafter, an extensive research on pre-existing ontologies encapsulating relative knowledge concepts was conducted. Upon the dilemma emerged concerning either the entire imports of concepts (where the majority of structures were needed) or manufacturing our own, we concluding in building custom concepts targeting precisely our objectives and linking afterwards where applicable. At the same time, we paid effort to keep a minimalistic approach in the design of the overall system. Based on the ontology already created, the aggregation of web content or content from official databases was populated in the knowledge base. Finally, rules that should diminish significantly the offered number of selections based on property constraints were implemented and incorporated into web Application Programming Interfaces (APIs).

The rest of the paper is structured as follows: Sect. 2 presents work which at some extent seems coherent to this paper. Sections 3 provides an overview of the framework and bestows the overall vision and motivation. In Sect. 4 elaboration on the inference, validation and consistency capabilities are exhibited, while in Sect. 5 fundamental reasoning functionalities relying on time and geo-spatial properties are showcased. In Sect. 6 quantitative and qualitative evaluation is displayed and, finally, Sect. 7 concludes our work. 


\section{Related Work}

Data about Cultural and Natural places are bound with their location information and time parameters. With the increasing amount of geospatial data being published online and the geographic information taking a crucial part in several central hubs on the Linked Data Web; geospatial semantics, geo-ontologies and semantic interoperability can have a key role in supporting publishing, retrieving, and reusing data while reducing the risks of misinterpretations [1,2]. Moreover, various semantic web technologies have been adapted for geospatial data, with progress in the effectiveness of the methods used in [3] with space and time having a key role for definition, organization and mutual interaction between concepts for knowledge engineering [4]. In similar approach, RDF models regarding spacetime events have been designed, that integrate spatial, temporal and semantic relations for capturing factors behind certain geographic changes [5].

Aggregating geospatial datasets into a single one is a challenging task. To tackle this problem semantic technologies were deployed so as to automate the geospatial data conflation process. By using ontology, RDF data conversion and a set of SWRL rules, one can produce a Points of Interest (POIs) dataset with reduced duplicates and improved accuracy [6]. Furthermore, the semantic ontological network graph (SONET) [7] is an ontological network to match categories across multiple heterogeneous sources of POIs of Volunteered Geographic Information (VGI) data. This ontological network advances the study of VGI data by enabling cross-platform analysis while it supports the use of POI data in land use mapping and population modeling applications. Deepening in the issue of heterogeneity since particularly cultural heritage consists of multiple resources which might include entities such as places, events, availability, and others that have special characteristics and might be connected with each other. Cultural$\mathrm{ON}$ is a suite of ontology modules, to model the principal elements identified in the cultural heritage data type classification. The result is a knowledge base consisting of semantic interconnections with also other data available in the Web to be exploited according to different tasks and users preferences [8].

Taking advantage of cultural assents and towards touristic recommendations, there are several ontology-based systems available. They provide personalized suggestions to users, based on user profiles and information concerning the suggested locations. The results are ranked based on profile assignment, content filtering and user feedback [9] or by ontology-based content analyzer, ontologybased profile learner, and ontology-based filtering component [10] or in the case of STAAR (Semantic Tourist information Access and Recommending) where algorithms take into consideration itinerary length and user interests [11].

\section{3 e-Tracer Framework}

In a world with liberty and convenience of locomotion, data can be aggregated in abundance, enabling the sector of personalized tourism to attribute with further enhancements to provide a more pleasant experience to the end-user, an effort also facilitated by e-Tracer [12], a national funded project. 


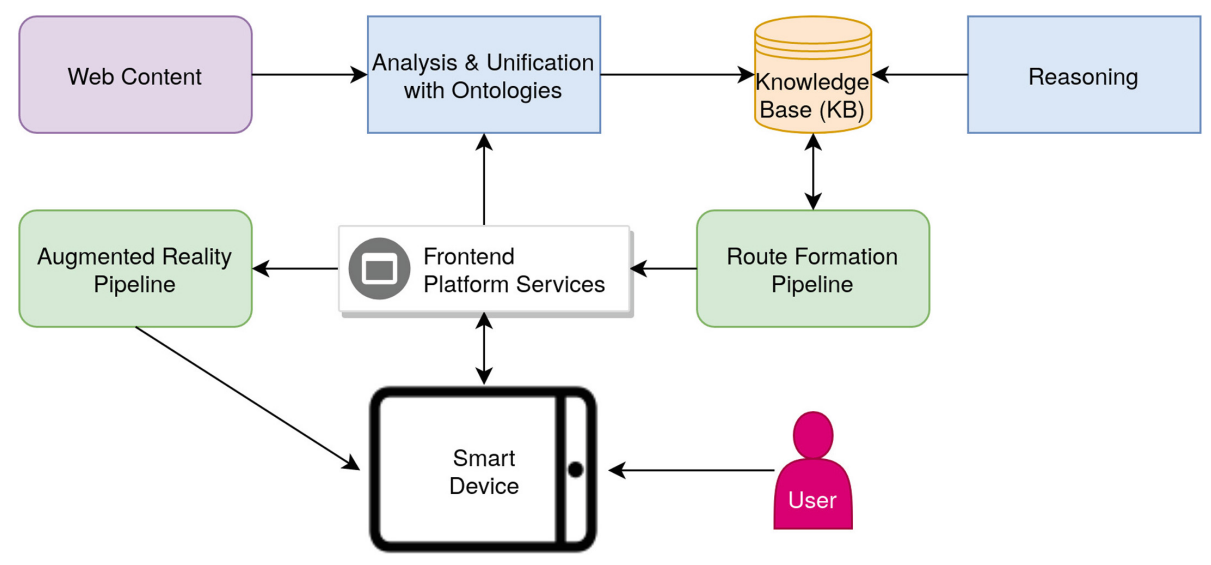

Fig. 1. The conceptual framework of e-Tracer

\subsection{Key Concepts and Vision}

The conceptual architecture of the platform is depicted in Fig. 1. Briefly, the Web Content involves data derived from official websites and open governmental databases. For the former the easIE framework [13] was used to scrap content, while for the later massive file exports sufficed. Furthermore, the Route Formation Pipeline expresses a complex algorithm to create routes, containing not only reasoning functionalities but also personalization based on similarity measures and graph algorithms such as the traveling salesman approaches, Dijkstra's shortest path and so on. Moreover, the Augmented Reality Pipeline encompasses search, identification, retrieval and display of objects on a Smart Device. Concluding, for aggregating information and presenting it to the User the Frontend Platform Services are responsible for.

Our work is focused on the semantics and aims to enrich e-Tracer with such capabilities. It acts as a semantic middleware, capturing, interlinking and serving results. This is materialized in the "Analysis \& Unification with Ontologies" component where dissimilar content is homogenized and stored locally in compliance with Resource Description Framework (RDF) triplets inside a Knowledge Base (KB). Additionally, it provides the semantic infrastructure to retrieve stored assets in an asynchronous on-demand manner to fulfil dynamic querying requirements. Furthermore, the reasoning occurs either automatically on each update of the $K B$ or by calling it as a service where data are reciprocated amphidromously. Concluding, it is obvious from Fig. 1 that the use of semantics stands in the core of the platform adding extra value and coordinating a majority of processes.

\section{2 e-Tracer Ontological Core Model}

Figure 2 illustrates the upper-level concepts of the e-Tracer hierarchical model where each differentiated arrow line is depicting a distinct type of connection 


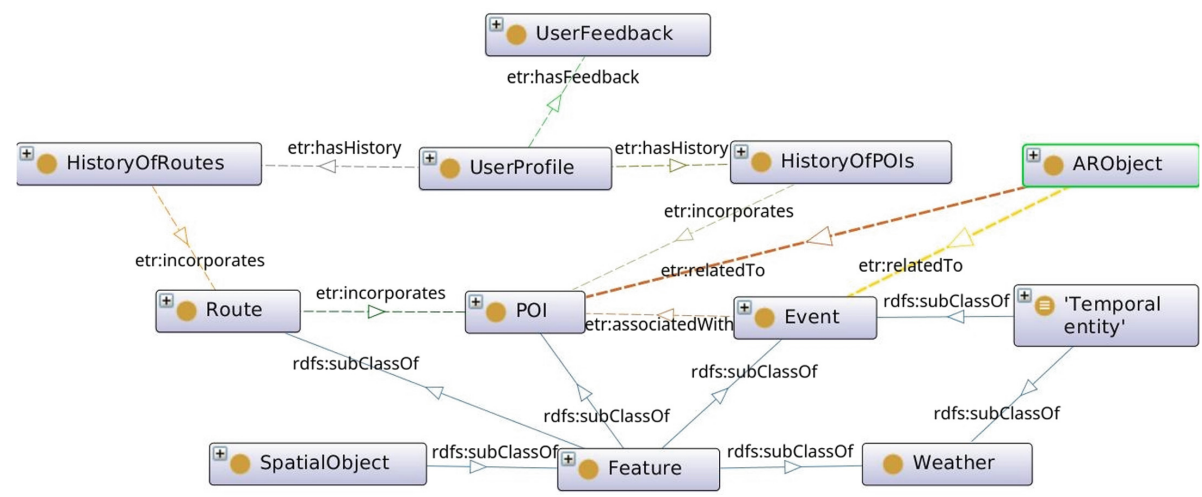

Fig. 2. The upper-level core concepts of the e-Tracer model and the semantic conjugation

and dotted lines implying customness. The conceptual model revolves around the notions of point of interest (POI), event, augmented reality object, spatial object, temporal entity, route, user profile, user feedback and weather. Each distinct connotation is potentially intertwined with others in manifold degrees, both at root and lower modeled levels, in a way that there should not be perceived in the overview graph such a phenomenon as an orphaned node when data increment efficiently and satisfactorily. Intuitively, entities will be linked with pre-existing online entities as soon as the core model reaches near its terminal configuration after numerous successive iterations. In the succeeding subsections we circumscribe extensively each key concept along with its semantic correlation with the rest.

Re-used Concepts. The concept of the Event is described both accurately and sufficiently by the ontology for linking open descriptions of events, LODE [14]. In its simplicity, it is explained more thoroughly in the next subsection along with how supplementary content was formed on top of this work to extend it.

The GeoSPARQL ontology [15], abiding by strictly to the Open Geospatial Consortium's standards, was shaped to represent and provide functionalities for objects possessing physical extent. In that aspect, as a "Feature" can be characterized anything from points in space to more intricate corporeal spatioformations such as polylines and acanonic, convex or non-convex, polygons. Noticeably, this supplementary property can be exploited to typify e-Tracer's concepts such as routes, POIs, events and furthermore the weather.

In conjunction with efforts towards the aforementioned localization, OWLTime [16] instils the nature of temporality into the e-Tracer ontology. Periodic and sporadic chronic intervals can be analytically specified and represented, spanning from time-limited events to recurring schedules of points of particular interest, as well as unfolding iterative events such as weather phenomena. Consequently, the fusion of space and time capacitates a thorough designation for 
when an entity is shifting through those dimensions, facilitating perpetual and unremitting knowledge monitoring of its evolutionary existence.

Finally, we took into consideration previous work regarding the User Profile concept and collected very few content from [17] as described further below and extended it according to our needs with additional properties and relations with other entities.

Points of Interest, Events and Routes. The necessity for differentiation between the POI and the Event relies on the logical assumption which dictates that an Event might occur at a POI, coinciding spatially at the exact same longitude and latitude, thus being associated with it or at a place which is not regarded as a $P O I$ at all, also containing coordinates unclaimed by any POI. On the contrary, a $P O I$ might or might not ever hold an event, without being self-defined by it in any case. In terms of OWL 2 semantics, this is defined as:

$$
\text { Route } \equiv \text { Feature } \sqcap \forall \text { includes.(POI } \sqcap \forall i s \text { AssociatedWith.Event })
$$

Regarding temporal discrepancies, we take into consideration that a $P O I$ is permanently established, mayhaps withholding a somewhat fixed schedule, whereas an Event is strictly time-limited and even if periodicity is witnessed, each resumption will be considered a distinct instance. Apart from the dimensional perspective, a qualitatively extensive context-based research, based on local governmental resolutions ${ }^{1,2,3,4,5}$, has been conducted so as to conclude to a classbased hierarchical categorization of eventual supported types of POIs and Events of interest.

Ultimately, the existence of an instance of the class Route solely depends on it incorporating either an instance of a $P O I$ or that of an Event at least, in a ranked manner which mandates the order of visit each Route suggests. Routes are being generated dynamically in a more compound integrated algorithm, which is out of the scope of this paper, due to the humongous population of potential options, thus not pre-processed or stored locally where the later occurs only after an end-user has completed successfully a proposed detour, on several occasions accompanied by an evaluation of his overall experience in the form of User Feedback.

User Profile, User Feedback and History of Usage. The User Profile construction encapsulates basic demographic information plus personal interests complemented by the end-user himself or potential impairments. Each instance is bound to possess a History of Routes and a History of POIs archiving each Route, and within, each POI the user has indeed paid a visit to, which is cross

\footnotetext{
${ }^{1}$ http://odysseus.culture.gr.

${ }^{2}$ http://listedmonuments.culture.gr.

${ }^{3}$ http://estia.minenv.gr/.

${ }^{4}$ http://www.minagric.gr.

${ }^{5}$ http://www.opengov.gr/.
} 
validated via the end-user's smart device geolocalization. Furthermore, the subclasses of User Feedback are related at a lower level with the historical assets of the POI and the Route of the ontology, rendering infeasible to submit a personal standardized evaluation about a spatial entity without a priori ratified attendance by the appropriate digital agent of the platform.

$$
\begin{gathered}
\text { UserProfile } \equiv \forall \text { hasFeedback.UserFeedback } \sqcap \forall \text { hasRouteHistory } \\
\text {.(HistoryOfRoutes } \sqcap \exists \text { hasRoute.Route) }
\end{gathered}
$$

Augmented Reality and Weather Features. The Augmented Reality Object intends to enclose a profusion of essential properties and describe thoroughly in a semantic way $2 \mathrm{D}, 2.5 \mathrm{D}$ and $3 \mathrm{D}$ objects. Unfortunately, there is a scarcity in modelled digital assets to the applied interests of e-Tracer, nevertheless, due to the purposeful adaptability of the system beyond fixed use cases, it was deemed vital to patronize such features. Pragmatically, such an object might only be related to spatial entities, potentially deriving implicit inferences from them according to each scenario. Moreover, the Weather in its simplicity is considered to hold, apart from self-explanatory data properties, both temporal and spatial attributes, achieving to monitor evolutionary weather phenomena across regions pertaining POIs or Events, eventually served to the end-user as plain information or taken into consideration in rule-based reasoning upon nominating routes.

\section{Inference and Validation}

\subsection{Implicit Relationships}

Extra logical assumptions are the outcome of blending native OWL 2 RL reasoning and manually constructed custom rules, where the prior relies on the OWL 2 RL profile semantics [18]. Sadly, OWL 2 is limited as it serves modeling only for instances related in a tree-like approach [19]. Our framework implements domain rules on top of the standard graphs in order to enunciate richer relations by the utilization of CONSTRUCT graph motifs, thus enabling the identification of valid inferences. For example, an Augmented Reality Object instance never contains information about its geolocalization but in our ontology is always attached to a spatial entity, which in turns contains coordinates that can be bequeathed to it via the suitable SPARQL CONSTRUCT query shown below:

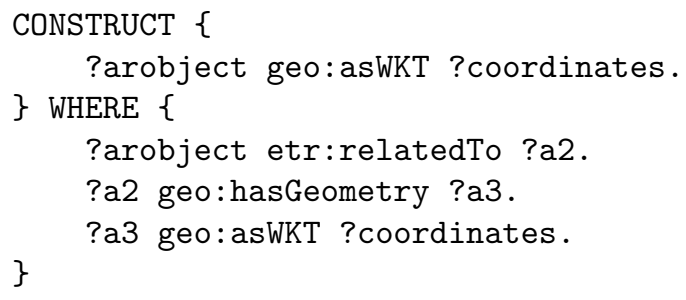




\subsection{Consistency and Validation Check}

The validation procedure guarantees the consistency of the framework along with the quality, both morphologic and syntactic. This scope is fulfilled through the usage of both custom SHACL [20] validation rules and native ontology consistency checking, always adhering to the closed-world paradigm. The latter manages validation by considering the semantics at TBOX, such as class disjointness, whereas the first discerns constraint contraventions like imperfect information or cardinality contradictions. For example, a SHACL shape representing a constraint which forces all POIs to contain exactly a single ID as a data property of type string is shown below:

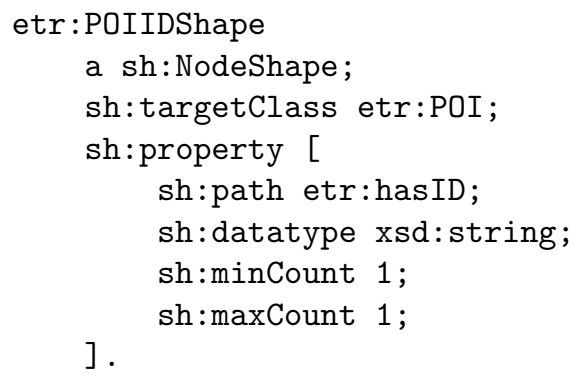

\section{Spatio-Temporal Rule-Based Reasoning}

Entire concepts were imported from well-known ontologies and were combined so as to administer especial properties to specific instances, thus conferring additional capabilities on ruled-based reasoning in order to succeed in a significant diminish in the pool of recommended selections which flow to posterior in-chain services with ultimate objective to deliver delightful route suggestions to the end-user.

The very essence of reasoning in e-Tracer relies on the meaningful restriction of proffered choices. It has already been stated explicitly that each place of interest withholds formal standardized coordinates nearby a major traffic network. On top of those coordinates, functions and APIs based on [15] were developed and utilized so as to estimate euclidean distances between interchanges of the initial route and the places of interest, realistically serving as a lower distance bound where at best case the euclidean distance equals the actual driving distance. In addition, the fixed traffic network speed limits facilitated the development of an algorithm about approximate calculation of the time needed to arrive from one place to another, also serving as a lower estimation bound.

The algorithms described above were fused into dynamic hybrid rules expressed in complex SPARQL queries, combining time and space dimensions and an additional boolean variable of accessibility impairment to showcase the true potential of complex rule-based reasoning. Furthermore, it is not obligatory to set all limitations at once for the API to function, e.g. sometimes we 
only mind for distance and not at all for time or accessibility. Consequently, by applying limitations when retrieving places of interest correlated to each interchange within the main route of the end-user, the options stand fewer than before based on logic and necessarily satisfy either default constraints or constraints set by the end-user himself. A sample SPARQL pseudocode applying time and space constraints is provided in Algorithm 1 where if input variables are set to zero the algorithm does not consider that variable for filtering at all:

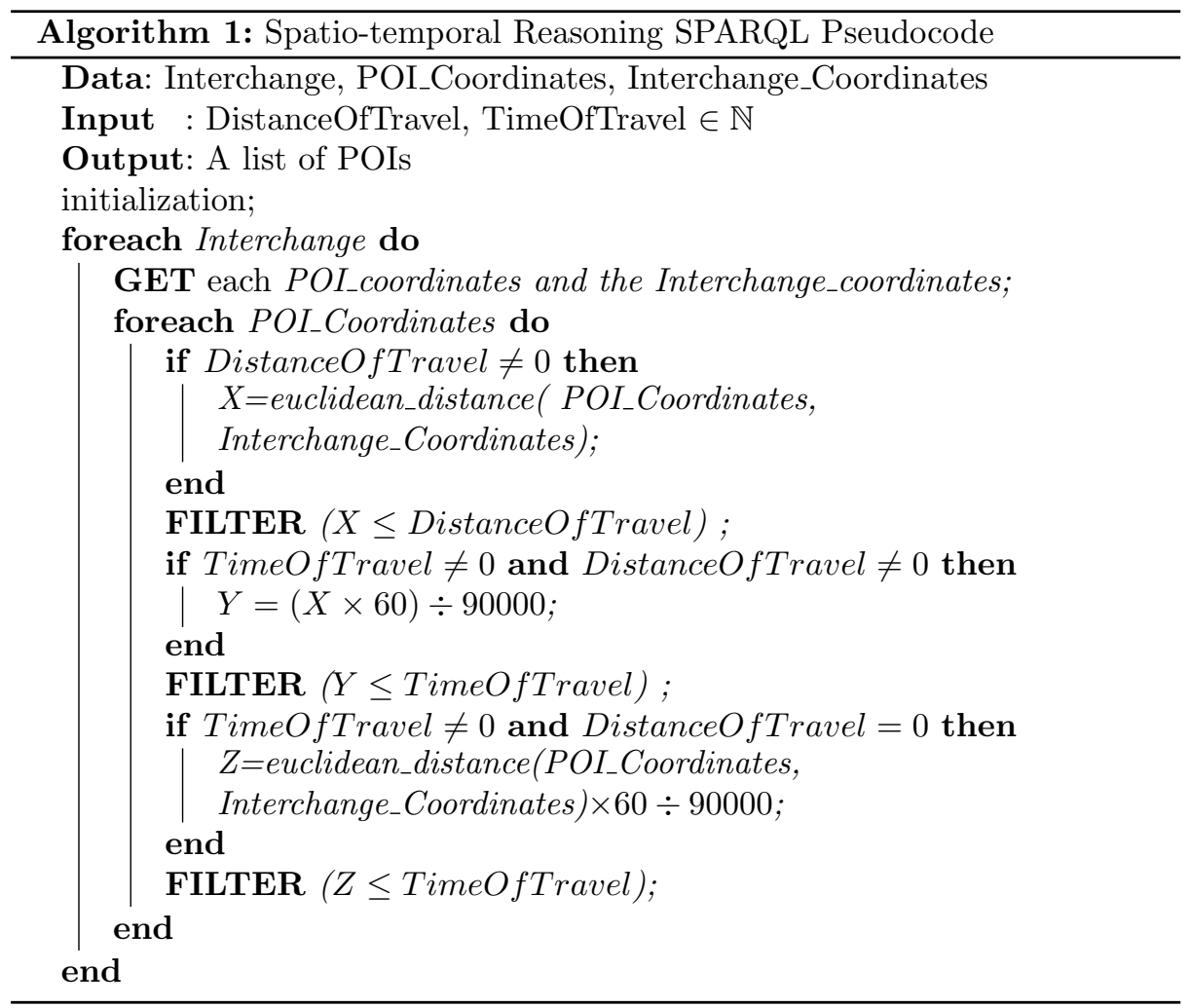

\section{Evaluation}

Currently, a user-centered evaluation stands infeasible as the pilots are due to commence in subsequent months, ipso facto we focalized in system-wise benchmarking. In that aspect, we demonstrate the population of the stored entities, shown in detail in Table 1. The triple store at our disposal is a GraphDB 9.1.1 Free Edition with currently stored 15286 triples which was deployed at a server with Ubuntu 18.04.4 LTS (Bionic Beaver) 64-bit operating system, an Intel Xeon(R) Silver $4108 \mathrm{CPU} @ 1.80 \mathrm{GHz} \times 32,62.5 \mathrm{~GB}$ of RAM and a Hard Disk Drive of 3.6 TB capacity. 
Table 1. The number of POIs and Events with (average) sum of properties for each, inside the knowledge base

\begin{tabular}{l|l|l}
\hline \#POIs \& Events & \#Properties & Avg. Properties per POI / Event \\
\hline 257 & 11627 & $\approx 45$ \\
\hline
\end{tabular}

Unfortunately, it is only anticipated to incline the evaluation towards the engineering response times in a manner where the bias is eliminated. All but one methods were manufactured as dynamic RESTful, thereby we ensured upon summoning that the variables on call conform to a uniformly distributed pseudogenerator with their range values spanning with equal probability of selection to all meaningful and valid content. All of them gratify the competency questions which were documented formerly of the creation of the e-Tracer ontology, a subset of which is showcased in Table 2, along with mean response times and standard deviations, elicited from 1000 executions for each.

Table 2. Exemplary competency questions

\begin{tabular}{|c|c|c|}
\hline \# & Question & $\begin{array}{l}\text { Mean (SD) } \\
\text { in msec }\end{array}$ \\
\hline Q1 & Retrieve all registered $P O I$ names with their respective IDs & $27 \pm 8$ \\
\hline Q2 & Retrieve all related properties to a pseudorandom $P O I$ & $19 \pm 10$ \\
\hline Q3 & $\begin{array}{l}\text { Retrieve all POI names with their IDs registered to a } \\
\text { pseudorandom interchange bound to pseudorandom time \& } \\
\text { distance constraints }\end{array}$ & $22 \pm 11$ \\
\hline Q4 & $\begin{array}{l}\text { Retrieve all related properties to POIs registered to a } \\
\text { pseudorandom interchange bound to pseudorandom time \& } \\
\text { distance constraints }\end{array}$ & $114 \pm 139$ \\
\hline Q5 & $\begin{array}{l}\text { Retrieve all related properties from multiple POIs registered to } \\
\text { pseudorandom multiple interchanges bound to pseudorandom } \\
\text { time \& distance constraints }\end{array}$ & $3277 \pm 4491$ \\
\hline
\end{tabular}

A simulation example of our approach is displayed in Fig. 3, while moving from point A to point $\mathrm{B}$, where all POIs retrieved from the interchange without any reasoning occurring stand 43 . On the contrary, it is conceived that the number of 5 POIs is noticeably less when the constraint of time is set to $30 \mathrm{~min}$ and that of the distance to $20 \mathrm{~km}$. 


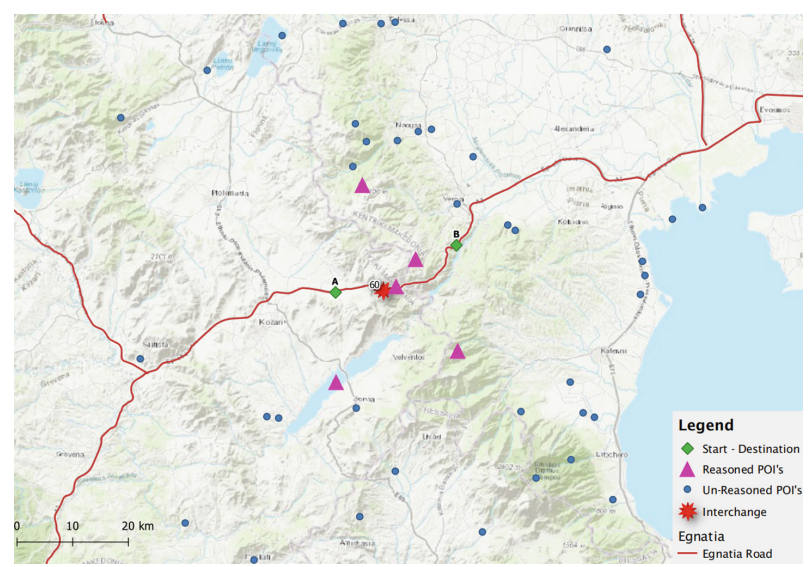

Fig. 3. Geographic map of simulation

\section{Conclusion and Future Work}

In this paper we presented an ontology-based framework for encapsulating and interlinking resources of cultural and natural nature towards the construction of suggestive enhanced routes. On top of the structured knowledge we practised rule-based reasoning based on spatial and temporal properties of the assets.

At the moment, the work featured is part of a synthesis of services, where dynamic routes are formed based only on the distinct unary level. Consequently, looking to the future, the next step is to exploit knowledge at a more aggregated level, such as applying reasoning at route level. Apart from reasoning, at final stages the resources of e-Tracer ought to be openly linked to other efforts, following the principles of Open Data $\&$ Linked Data.

The evaluation plan at a cultural level will be orchestrated by Piraeus Bank Group Cultural Foundation whereas at environmental level Axios - Loudias - Aliakmonas Delta, Koronia-Volvi and Pamvotis lakes protected area management bodies are responsible for. The platform encapsulates the Egnatia Motorway axis for pilots and content provided by the prior organizations. Finally, the assessment of the prototypes will be conducted during the pilot tests applied to 2 collaborating museums (the silversmithing museum and the silk museum) and at least 3 areas of environmental interest.

Acknowledgements. This work is co-financed by the European Union and Greek national funds via the Operational Program Competitiveness, Entrepreneurship and Innovation, under the call RESEARCH-CREATE-INNOVATE (project code: T1E $\Delta \mathrm{K}-$ 00410). 


\section{References}

1. Janowicz, K., Scheider, S., Pehle, T., Hart, G.: Geospatial semantics and linked spatiotemporal data-Past, present, and future. Semant. Web 3(4), 321-332 (2012)

2. Homburg, T., et al.: Interpreting heterogeneous geospatial data using semantic web technologies. In: Gervasi, O., et al. (eds.) ICCSA 2016. LNCS, vol. 9788, pp. 240-255. Springer, Cham (2016). https://doi.org/10.1007/978-3-319-42111-7_19

3. Zhao, T., Zhang, C., Wei, M., Peng, Z.-R.: Ontology-based geospatial data query and integration. In: Cova, T.J., Miller, H.J., Beard, K., Frank, A.U., Goodchild, M.F. (eds.) GIScience 2008. LNCS, vol. 5266, pp. 370-392. Springer, Heidelberg (2008). https://doi.org/10.1007/978-3-540-87473-7_24

4. Janowicz, K.: The role of space and time for knowledge organization on the semantic web. Semant. Web 1(1, 2), 25-32 (2010)

5. Fan, J., Stewart, K.: Modeling and reasoning about geospatial event dynamics using semantic web technologies. In: SDW@ GIScience, pp. 17-25 (2016)

6. Yu, F., McMeekin, D.A., Arnold, L., West, G.: Semantic web technologies automate geospatial data conflation: conflating points of interest data for emergency response services. In: Kiefer, P., Huang, H., Van de Weghe, N., Raubal, M. (eds.) LBS 2018. LNGC, pp. 111-131. Springer, Cham (2018). https://doi.org/10.1007/978-3-31971470-7_6

7. Palumbo, R., Thompson, L., Thakur, G.: SONET: a semantic ontological network graph for managing points of interest data heterogeneity. In: Proceedings of the 3rd ACM SIGSPATIAL International Workshop on Geospatial Humanities, pp. 1-6, November 2019

8. Lodi, G., et al.: Semantic web for cultural heritage valorisation. In: Hai-Jew, S. (ed.) Data Analytics in Digital Humanities. MSA, pp. 3-37. Springer, Cham (2017). https://doi.org/10.1007/978-3-319-54499-1_1

9. Alonso, K., et al.: Ontology-based tourism for all recommender and information retrieval system for interactive community displays. In: 2012 8th International Conference on Information Science and Digital Content Technology (ICIDT 2012), vol. 3, pp. 650-655. IEEE, June 2012

10. Bahramian, Z., Abbaspour, R.A.: An ontology-based tourism recommender system based on spreading activation model. International Archives of the Photogrammetry, Remote Sensing \& Spatial Information Sciences, 40 (2015)

11. Cao, T.D., Phan, T.H., Nguyen, A.D.: An ontology based approach to data representation and information search in smart tourist guide system. In: 2011 Third International Conference on Knowledge and Systems Engineering, pp. 171-175. IEEE, October 2011

12. Stathopoulos, E.A., et al.: Smart discovery of cultural and natural tourist routes. In: IEEE/WIC/ACM International Conference on Web Intelligence-Companion Volume, pp. 208-214, October 2019. https://doi.org/10.1145/3358695.3361105

13. Gkatziaki, V., Papadopoulos, S., Mills, R., Diplaris, S., Tsampoulatidis, I., Kompatsiaris, I.: easIE: easy-to-use information extraction for constructing CSR databases from the web. ACM Trans. Internet Technol. (TOIT) 18(4), 1-21 (2018)

14. Shaw, R., Troncy, R., Hardman, L.: LODE: linking open descriptions of events. In: Gómez-Pérez, A., Yu, Y., Ding, Y. (eds.) ASWC 2009. LNCS, vol. 5926, pp. 153-167. Springer, Heidelberg (2009). https://doi.org/10.1007/978-3-642-108716_11

15. Battle, R., Kolas, D.: Geosparql: enabling a geospatial semantic web. Semant. Web J. 3(4), 355-370 (2011) 
16. Hobbs, J.R., Pan, F.: Time ontology in OWL. W3C working draft, 27, 133 (2006)

17. Maria, G., Akrivi, K., Costas, V., George, L., Constantin, H.: Creating an ontology for the user profile: method and applications. In: Proceedings AI* AI Workshop RCIS (2007)

18. Motik, B., Grau, B.C., Horrocks, I., Wu, Z., Fokoue, A., Lutz, C.: OWL 2 web ontology language profiles. W3C recommendation, 27, 61 (2009)

19. Motik, B., Cuenca Grau, B., Sattler, U.: Structured objects in OWL: representation and reasoning. In: Proceedings of the 17th International Conference on World Wide Web, pp. 555-564, April 2008

20. Knublauch, H., Kontokostas, D.: Shapes Constraint Language (SHACL), W3C Recommendation. World Wide Web Consortium (2017) 